\title{
Topical Cocaine Hydrochloride
}

National Cancer Institute

\section{Source}

National Cancer Institute. Topical Cocaine Hydrochloride. NCI Thesaurus. Code C104004.

A topical formulation of the hydrochloride salt of the tropane alkaloid cocaine, with local anesthetic activity. Upon topical application of the cocaine hydrochloride solution to nasal mucous membranes, cocaine reversibly binds to and blocks the voltage-gated sodium channels in the neuronal cell membranes. By stabilizing neuronal membranes, cocaine inhibits the initiation and conduction of nerve impulses and produces a reversible loss of sensation. 